\title{
The role of physiological assessment in team selection with special reference to rowing
}

\author{
Yiannis Koutedakis BSc, MA
}

Senior Exercise Physiologist, British Olympic Medical Centre, Northwick Park Hospital, Harrow, Middlesex, UK and former National Coach for Junior Rowing, Amateur Rowing Association

Successful competitive performance is particularly associated with three main attributes: skill, appropriate psychological attitude and physical fitness of the individual competitor.

It has long been established that no single parameter measures physical fitness, which is a composite factor varying with each sport. Such 'fitness' may contain a number of different elements including maximal oxygen uptake, local muscle endurance, muscle strength, and body composition ${ }^{1}$. Laboratory assessments on male British Olympic competitors in various sports have revealed maximal oxygen uptake values ranging from 40 to $88 \mathrm{ml} . \mathrm{kg}^{-1} \mathrm{~min}^{-1}$, body fat measurements ranging from 6 to 29 per cent, and isokinetic strength of the knee extensors ranging from 40 to 250 foot/pounds on angular velocity of $60^{\circ} \mathrm{sec}^{-1}$.

In general, physiological assessment of elite competitors may be used to determine physical working capacity and predict physiological potential, to aid in planning and appropriately modifying training programmes and competitive tactics, to elucidate symptoms related to fatigue and underperformance, and to assist in team selection, particularly at national level. These factors should eventually result in optimizing the available training time of both competitors and coaches in their effort to improve performance.

Methods of selecting national teams vary not only among countries, but also among sports. Selectors for individual sports such as athletics, wrestling, and judo, would appear to have fewer problems, since participants in these events usually gain representation during objective trials on an individual 'knock-out' basis. This process of selection is sometimes used for team sports, but most national sporting associations prefer to choose the required number of team members for a particular sport from among their most outstanding individual athletes.

Most countries use selection committees to choose their representative national rowing teams. Prior to any major international competition, such a committee is responsible for putting together the best rowers from the various crews. The method of 'seat-racing' (a series of inter-crew races with single seat-by-seat

(C) 1989 Butterworth \& Co (Publishers) Ltd 0306-3674/89/010051-02\$03.00 member changes) has also been recently used by certain countries including the USA and UK.

Dissatisfaction expressed by selectors, coaches and particularly rowers has prompted the rowing authorities to seek more objective criteria for selection, and to engage an unbiased source of assistance in the form of laboratory assessment. One of the early recorded cases of crew selection based largely on the outside source of laboratory physiological measurements, concerned two members of the 1968 Olympic gold medal New Zealand coxed four. This led to the physiological profiling of elite oarsman, where high levels of muscular strength and oxygen consumption have been considered essential for success in national and international rowing ${ }^{2}$. However, in deciding between rowing candidates of apparently similar aerobic capacity, the rower with the higher anaerobic threshold should theoretically be capable of performing closer to his or her maximum before being limited by the buildup of lactic acid. Furthermore, as serum lactate begins to rise, tolerance of lactate in the exercising muscle may be of critical importance ${ }^{3}$.

A systematic approach should be used to analyse factors limiting an athlete's performance. Serial measurements, preferably three or four times a year, provide an opportunity to reduce a large group of rowers (or other competitors) to a workable number and to assess the specific benefits of training. Repeated laboratory measurements can determine rowers' mechanical efficiency (the ratio between oxygen cost and power output), an important parameter for high level performance 4 .

For both team selection and physiological monitoring, laboratory simulation of the sport action is important in obtaining values which truly reflect the potential of the individuals. For this purpose, appropriate equipment (ergometers and/or dynamometers) and carefully planned test protocols are essential.

Cycle ergometry was the main testing mode in earlier sports studies. Physiological responses, including maximal aerobic capacities, were lower than during treadmill running. However, treadmill running may be unsuitable for assessing rowers since rowing is not a weight-bearing exercise and, moreover, rowing is a four-limb activity. Both friction-loaded and air-resistance rowing ergometers have been introduced for as- 


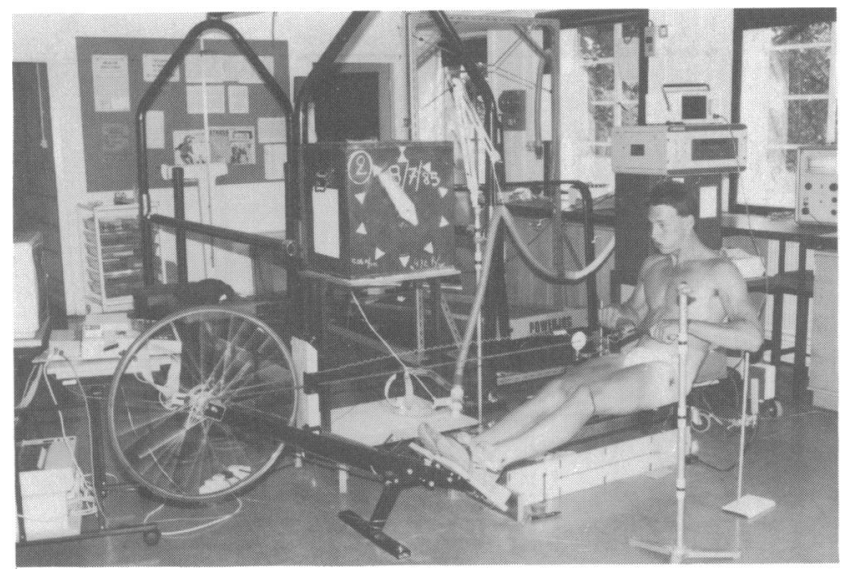

Figures 1-3. Athletes undergoing physiological assessment

sessing physical working capacity. Unfortunately, the resistance characteristics of friction-loaded ergometers do not provide an adequate simulation of rowing ${ }^{5}$, whereas the advantage of the air-resistance machine and its close simulation to rowing action in a boat has been strongly supported ${ }^{6}$. An increase in power of about 30 per cent is needed to raise the boat's speed by 10 per cent and this is simulated satisfactorily by the air-braked ergometer.

Three different test protocols can be used for cardiorespiratory assessment of rowers:

(i) An all-out continuous test where the subjects have to perform maximally for a predetermined period. This protocol prevents acquisition of some important information (such as anaerobic threshold data). Changes in aerobic metabolism are reflected both in the maximal oxygen consumption and in the way in which this is reached (in terms of the slope of the oxygen uptake curve).

(ii) A discontinuous incremental protocol where blood sampling techniques may be applied. This is less commonly used as it is less satisfactory for rowers to be tested maximally under discontinuous conditions when their sporting effort is normally continuous.

(iii) A continuous incremental protocol which permits the gradual elevation of cardiorespiratory and metabolic responses to the point at which exercise intensity exceeds the capacity of the available oxygen to sustain the load (the anaerobic threshold). It then allows the athlete to continue exercising in stepwise progression beyond the anaerobic threshold until his maximum oxygen consumption has been attained ${ }^{7}$.

To conclude, physiological measurements may significantly contribute to team selection, but results will be misleading unless sport-specific procedures are followed. That component of team selection which is based on laboratory assessments should include repeated data collection sessions, thus allowing progress in the key physiological parameters to be monitored.
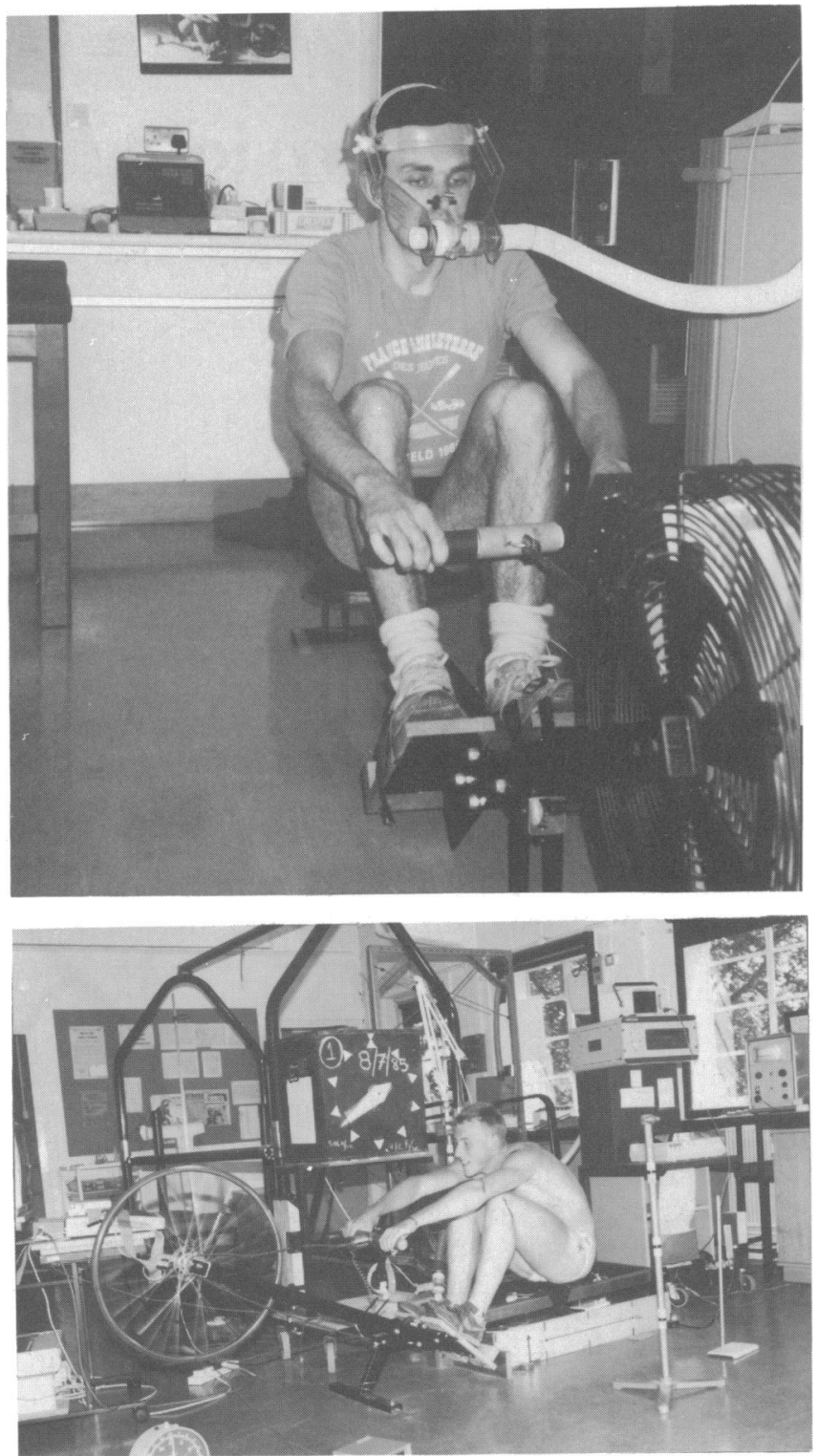

\section{Acknowledgement}

The author wishes to thank Mr Bernard Carpenter for his help.

\section{References}

1 Sharp, N.C.C. Fitness and its measurement The Practitioner 1988, 232 (8), 974-976

2 Hagerman, F.C. Applied physiology of rowing Sports Medic 1984, 1, 303-326

3 Koutedakis, Y. and Sharp, N.C.C. A modified Wingate Test for measuring anaerobic work of the upper body in junior rowers Brit J Sports Med 1986, 20, 153-156

4 Secher, N.H. The physiology of rowing J Sports Sci 1983, 1, 23-53

5 Koutedakis, Y. Ergometry - use of rowing ergometer Rowing 1987, 30 (336), 17-18

6 Hagerman, F.C., Lawrence, R.A. and Mansfield, M.C. A comparison of energy expenditure during rowing and cycling ergometry Med Sci Sports Exerc 1988, 20 (5), 479488

7 Mahler, D.A., Andrea, B.E. and Andresen, D.C. Comparison of 6-min 'all-out' and incremental exercise tests in elite oarsmen Med Sci Sports Exer 1984, 16, 567-571 